Actualidades en Psicología, 33(127), julio-diciembre 2019, 113-141 http://revistas.ucr.ac.cr/index.php/actualidades

ISSN 2215-3535

DOI: $10.15517 /$ ap.v33i127.34975

Recibido: 24 de octubre de 2018

Aceptado: 13 de noviembre de 2019

\title{
Hacia un envejecimiento saludable: una revisión sistemática sobre la música y el ejercicio físico como factores moduladores
}

\section{Towards a Healthy Aging: A Systematic Review about Music and Physical Exercise as Modulating Factors}

\author{
Veronika Diaz Abrahan ${ }^{1}$ \\ Maximiliano Bossio ${ }^{2}$ \\ Nadia Justel ${ }^{3}$ \\ ${ }^{1,2,3}$ Laboratorio Interdisciplinario de Neurociencia Cognitiva (LINC). Centro de Estudios \\ Multidisciplinarios en Sistemas Complejos y Ciencias del Cerebro (CEMSC ${ }^{3}$ ). Instituto de Ciencias Físicas (ICIFI). \\ Escuela de Ciencia y Tecnología (ECyT). Universidad Nacional de San Martin (UNSAM), Argentina \\ ${ }^{1,3}$ Consejo Nacional de Investigaciones Científicas y Tecnológicas, Argentina \\ ${ }^{1,3}$ Universidad Nacional de Córdoba, Argentina \\ ${ }^{1,2}$ Universidad de Buenos Aires, Argentina
}

\begin{abstract}
Resumen. El envejecimiento se define como un conjunto de procesos que conllevan cambios biológicos, fisiológicos, psicosociales y funcionales, caracterizados por la manifestación de un deterioro cognitivo gradual y progresivo. Actualmente, hay un fuerte interés en identificar qué factores promueven un envejecimiento cognitivo saludable, así como en identificar qué intervenciones y estrategias brindan protección contra enfermedades neurodegenerativas relacionadas con la edad. Objetivo. El objetivo de la presente revisión sistemática es presentar las investigaciones que usan a la música o la actividad física como intervenciones que modulan aspectos fisiológicos y cognitivos del proceso de envejecimiento. Método. Se incluyeron estudios desde el año 2007 al 2018, cuya búsqueda se realizó en bases de datos (Redalyc, Scielo, Science Direct, PubMed, Taylor, \& Francis) y en la que se obtuvieron 34 artículos empíricos (17 relacionados con música y 17 con ejercicio físico). Conclusión. La principal conclusión es que se halla un desempeño cognitivo mejorado en los adultos mayores fruto de este tipo de intervenciones.
\end{abstract}

Palabras clave. Envejecimiento, música, actividad física.

Abstract. Aging is defined as a set of biological processes genetically determined, that are characterized by the manifestation of gradual and progressive cognitive deterioration. Currently, there is a strong interest to identify which factors promote healthy cognitive aging, and the interventions and strategies that protect against age-related neurodegenerative diseases. The aim of this systematic review is to outline non-pharmacological interventions that included music or physical activity programs for older adults. Studies published between the years of 2007 and 2018 in major databases (Redalyc, Scielo, Science Direct, PubMed, Taylor, \& Francis) were included in this review, obtaining 34 empirical articles (17 related with music and 17 with physical activity). The main conclusion is that an enhanced cognitive performance is found in older adults who were exposed to these interventions.

Keywords. Aging, music, physical activity.

${ }^{1}$ Veronika Díaz Abrahan. Laboratorio Interdisciplinario de Neurociencia Cognitiva (LINC). Centro de Estudios Multidisciplinarios en Sistemas Complejos y Ciencias del Cerebro (CEMSC ${ }^{3}$. Instituto de Ciencias Físicas (ICIFI). Escuela de Ciencia y Tecnología (ECyT). Universidad Nacional de San Martin (UNSAM). Consejo Nacional de Investigaciones Científicas y Tecnológicas (CONICET). Universidad Nacional de Córdoba (UNC), Argentina. Dirección Postal: Labo Cluster, 1 Piso, Of. 18, 25 de Mayo 1169. (1650) San Martín, Buenos Aires. Argentina. E-mail: abrahanveronika@conicet.gov.ar ${ }^{2}$ Maximiliano Bossio. Laboratorio Interdisciplinario de Neurociencia Cognitiva (LINC). Centro de Estudios Multidisciplinarios en Sistemas Complejos y Ciencias del Cerebro (CEMSC ${ }^{3}$ ). Instituto de Ciencias Físicas (ICIFI). Escuela de Ciencia y Tecnología (ECyT). Universidad Nacional de San Martin (UNSAM). Universidad de Buenos Aires (UBA), Argentina. E-mail: m.alejobossio@gmail.com

${ }^{3}$ Nadia Justel. Laboratorio Interdisciplinario de Neurociencia Cognitiva (LINC). Centro de Estudios Multidisciplinarios en Sistemas Complejos y Ciencias del Cerebro (CEMSC ${ }^{3}$. Instituto de Ciencias Físicas (ICIFI). Escuela de Ciencia y Tecnología (ECyT). Universidad Nacional de San Martin (UNSAM).Consejo Nacional de Investigaciones Científicas y Tecnológicas (CONICET). Universidad Nacional de Córdoba (UNC), Argentina. E-mail: nadiajustel@conicet.gov.ar

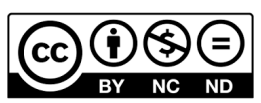

Esta obra está bajo una licencia de Creative Commons Reconocimiento-NoComercial-SinObraDerivada 4.0 Internacional. 


\section{Introducción}

La neurociencia cognitiva del envejecimiento es una rama disciplinar en pleno auge, que tiene como objeto de estudio el análisis de los factores y los mecanismos que influyen en el envejecimiento a nivel neuroanatómico y cognitivo (Díaz \& Pereiro, 2018). Con el continuo aumento de la esperanza de vida, el envejecimiento cerebral y cognitivo se ha convertido en una cuestión clave en ciencia, por lo que en los últimos años se han realizado investigaciones que estudian cuáles son aquellas funciones cognitivas que se encuentran afectadas en un envejecimiento saludable o no patológico (Grandi \& TirapuUstárroz, 2017).

El envejecimiento se define como un proceso gradual a lo largo de la vida que conlleva cambios biológicos, fisiológicos, psicosociales y funcionales, los cuales resultan en consecuencias variadas que se asocian a interacciones dinámicas entre las personas y el ambiente que los rodea (Organización de los Estados Americanos, 2015). En este sentido, el ambiente y el estilo de vida de los sujetos (dieta, educación, actividad, física, experiencias creativas, entre otras) serían factores relevantes en el proceso de envejecimiento. Por otra parte, y desde una perspectiva focalizada en aspectos biológicos, la Organización Mundial de la Salud define al envejecimiento como el conjunto de procesos genéticamente determinados, caracterizados por la manifestación de un deterioro funcional progresivo y generalizado, que ocasiona una pérdida de la respuesta adaptativa y un aumento del riesgo de contraer diversas enfermedades asociadas a la edad (OMS, 2017). La población definida como adulto mayor (AM) son aquellos sujetos que poseen una edad igual o superior a 60 años en países sub-desarrollados y 65 años en los desarrollados (OMS, 2017).

Las diferencias interindividuales con respecto al desempeño cognitivo aumentan con la edad y las consecuencias clínicas de la atrofia cerebral relacionada con el envejecimiento difieren de un individuo a otro (Fauvel et al., 2014). En relación a ello, se presentan dos conceptos destacados dentro de la temática: la reserva cerebral y la reserva cognitiva (Stern et al., 2018). El primero se basa en el aumento del volumen de la materia gris que contrarresta la atrofia y el retraso de la aparición de los primeros síntomas clínicos del envejecimiento (Getz, 2011). Por otra parte, pero en la misma línea, la reserva cognitiva hace referencia a los mecanismos neurocognitivos compensatorios que intentan maximizar el rendimiento frente al daño cerebral mediante el uso de estructuras cerebrales o redes no comprometidas.

De esta forma, actualmente hay un fuerte interés en identificar qué factores promueven un envejecimiento cognitivo saludable, así como también qué intervenciones y estrategias brindan protección contra enfermedades neurodegenerativas relacionadas con la edad (Balbag, Pedersen, \& Gatz, 2014). En esta línea, la calidad de las reservas (cerebral y cognitiva) está influida por características ambientales (Foubert-Samier et al., 2010), como por ejemplo el nivel educativo (Evans et al., 1993), el tipo y nivel ocupacional (Adam, Bonsang, Grotz, \& Perelman, 2013), habilidades intelectuales, interacciones sociales o actividades de la vida diaria (Seinfeld, Figueroa, Ortiz-Gil, \& SanchezVives, 2013; Wang, Xu, \& Pei, 2012). En este marco, la música y la actividad física son identificadas como factores ambientales que previenen o retrasan el déficit cognitivo producto del envejecimiento (Erickson et al., 2011; Hanna-Pladdy, \& Gajewski 2012; 
Schneider, Hunter, \& Bardach, 2018). Por ejemplo, en los últimos años, los estudios sugieren que el entrenamiento musical y ciertas actividades musicales acompañan un desarrollo cognitivo saludable a lo largo de la vida (Hanna-Pladdy \& Gajewski, 2012), así como también pueden mitigar el deterioro cognitivo que emerge en el proceso de envejecimiento (Wang \& Schlaug, 2010), debido al compromiso neuroanatómico y funcional que presenta la música (Justel \& Díaz Abrahan, 2012). Por otra parte, la actividad física se ha vuelto un foco de creciente interés para las investigaciones, ya que se identifica como un posible factor ambiental que favorecería el desarrollo y mantenimiento de las funciones cognitivas en diversas poblaciones (Chen, Yan, Yin, Pan, \& Chang, 2014; Hayes, Forman, \& Verfaellie, 2014; Hwang et al., 2016; Lee et al., 2014). Dicha ganancia se sumaría a los múltiples beneficios que normalmente se asocian a una vida comprometida con la actividad física, tales como el fortalecimiento y desarrollo de las estructuras musculares y óseas, y un mantenimiento saludable de los sistemas cardíaco y vascular (Hogan, Mata, \& Carstensen, 2013). Por lo expuesto hasta el momento, el objetivo de este trabajo de revisión sistemática es poner en evidencia aquellas investigaciones que documentan ventajas de la música y el ejercicio físico sobre aspectos cognitivos de los adultos mayores, durante el proceso de envejecimiento tanto saludable como patológico, y mostrar, a su vez, las limitaciones de estas investigaciones.

\section{Método}

\section{Diseño}

Se realizó una revisión sistemática de la literatura sobre la utilización de dos factores ambientales, la música y la actividad física, como posibles intervenciones ambientales no farmacológicas que modularían los procesos fisiológicos y cognitivos del envejecimiento. El grupo etario correspondió a AM ( $\geq 60$ años en países subdesarrollados; $\geq 65$ años en los desarrollados), que atraviesan un envejecimiento saludable o patológico.

\section{Muestra}

Treinta y cuatro artículos empíricos, 17 relacionados con música y 17 con ejercicio físico, fueron seleccionados luego de realizar la búsqueda bibliográfica en bases de datos científicas indexadas. Los criterios de inclusión fueron estudios empíricos que: (a) abordaran a la música y/o la actividad física como intervención/tratamiento; (b) contaran con la participación de AM dentro de la población de interés (cognitivamente saludables o con algún tipo de deterioro cognitivo); (c) dentro de su diseño experimental, cuasi experimental y estudios de cohortes, incluyeran variantes metodológicas para intervenciones musicales o respectivas a la actividad física deportiva; (d) contuvieran las palabras clave para los términos relevantes en inglés; y (e) estuvieran comprendidos entre los años 2007 a 2018.

\section{Procedimento}

Se utilizaron artículos recuperados de diversos motores de búsqueda, proceso que se llevó a cabo entre los meses de abril y octubre de 2018, sin emplear un protocolo específico de revisión. La búsqueda se realizó en diferentes bases de datos: Redalyc, Scielo, Science Direct, PubMed y Taylor \& Francis, junto a la recuperación de fuentes secundarias extraídas de los artículos seleccionados. 
La primera búsqueda estuvo guiada por las siguientes palabras clave: aging, AND/OR music AND/OR physical activity, y se obtuvieron aproximadamente 5385 resultados. De ese total de artículos, se realizó una segunda selección teniendo en cuenta las siguientes palabras clave específicas: cognitive reserve, AND/OR music training, AND/OR physical activity, delimitando la cantidad a 1937 artículos. A través de una tercera selección, basada en la lectura del resumen de cada publicación, se preseleccionaron 87 trabajos de los cuales 53 fuentes bibliográficas fueron posteriormente extraídas dado que poseían una fecha de publicación dentro del periodo 2007-2018. Finalmente, se descartaron 20 artículos, los cuales no cumplían con los criterios de inclusión. De esta forma, la muestra final estuvo conformada por 33 artículos principales (ver Tabla 1 y Tabla 2). Los estudios fueron interpretados independientemente por cada autor de este trabajo utilizando un enfoque cualitativo, el cual exige identificar categorías teóricas que son derivadas de los datos mediante la utilización de un método comparativo constante, teniendo en cuenta las siguientes variables de análisis: (a) tipo de muestra; (b) tipo de intervención (entrenamiento o intervención de largo, o mediano plazo o focal); (c) tipo de población (si se trata de AM con/sin deterioro cognitivo y género de la muestra); (d) comparaciones inter/intra-grupales; (d) variable dependiente analizada en cada estudio; (d) evaluación realizada; y (e) desempeños diferenciales de los individuos con base en la presencia o ausencia de las variables de interés. En la Figura 1 se observa el diagrama de flujo de la selección de los artículos.

\section{Resultados}

\section{Música y adultos mayores}

En los siguientes apartados se presentarán estudios que dan cuenta de cómo el entrenamiento musical a largo plazo, así como intervenciones focales, pueden afectar el envejecimiento de los sujetos.

\section{Entrenamientos de largo plazo}

Este apartado se centrará en las comparaciones entre músicos y no músicos, ya que las experiencias tempranas de aprendizaje musical influyen en las habilidades perceptivas, motoras y cognitivas de los sujetos. Las investigaciones que brindan evidencia a la temática son aquellas que evalúan las funciones cognitivas de AM músicos que iniciaron su aprendizaje musical a edades tempranas. Por ejemplo, Watanabe, Savion-Lemieux y Penhune (2007) investigaron la sincronicidad rítmica, evaluada con una tarea de tiempo de reacción en serie, de adultos que comenzaron sus entrenamientos antes (músicos tempranos) o después de los 7 años de edad (músicos tardíos). Los resultados mostraron que los músicos tempranos rindieron significativamente mejor que los músicos tardíos. Estos datos apoyan la idea de que habría un período sensible en la infancia donde el entrenamiento motor a través de la práctica musical trae aparejado beneficios duraderos para el rendimiento cognitivo. Sin embargo, este último punto parece ser un tema controvertido, ya que existen estudios que no encuentran diferencias significativas en cuanto a la edad de adquisición de contenidos musicales de los músicos (Hanna-Pladdy \& MacKay, 2011).

Para arrojar luz acerca del tema del período óptimo para el aprendizaje musical, Fauvel et al. (2014) realizaron dos estudios los cuales evaluaban, por un lado, la relación entre 


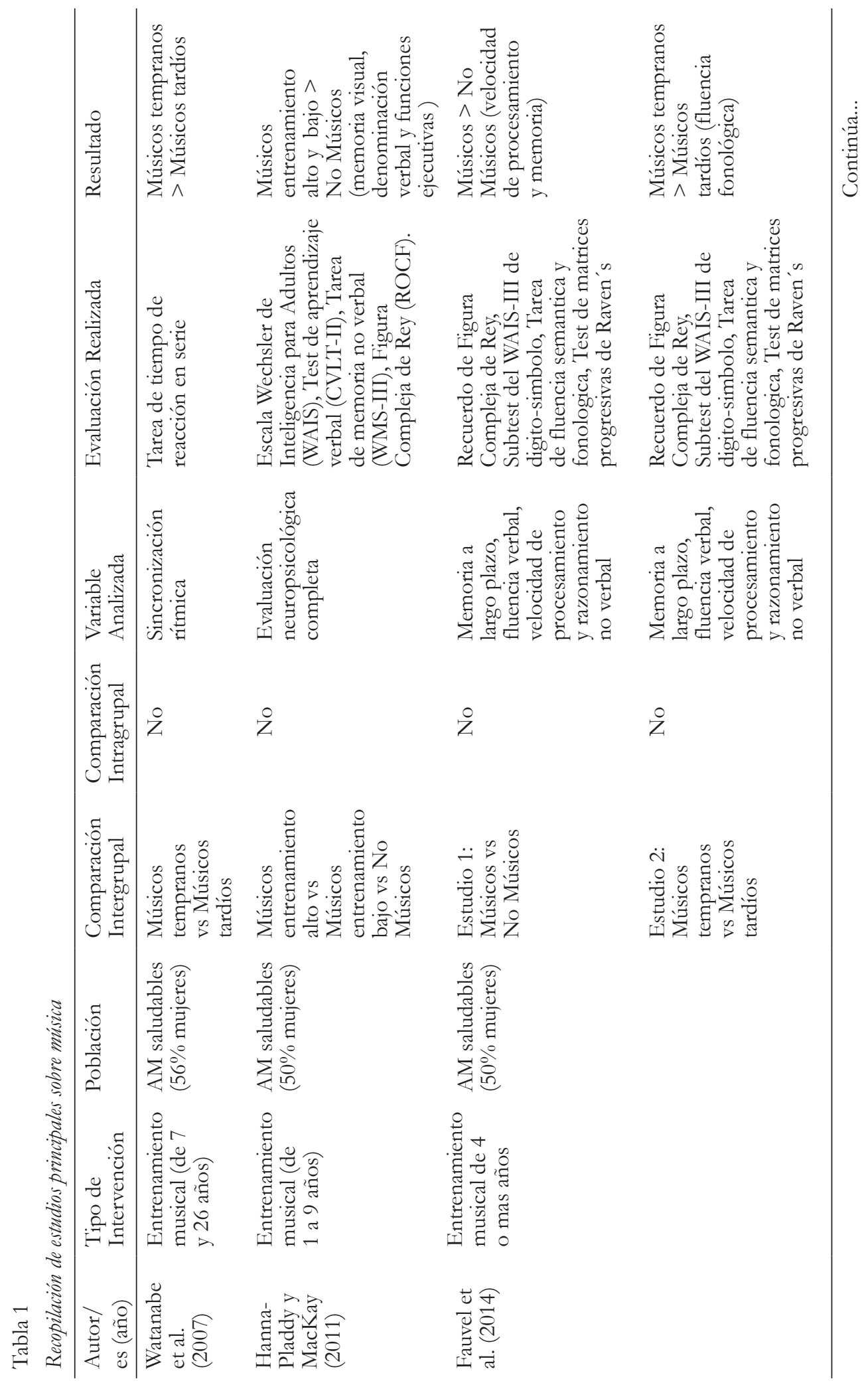

Actualidades en Psicología, 33(127), julio-diciembre 2019, 113-141 


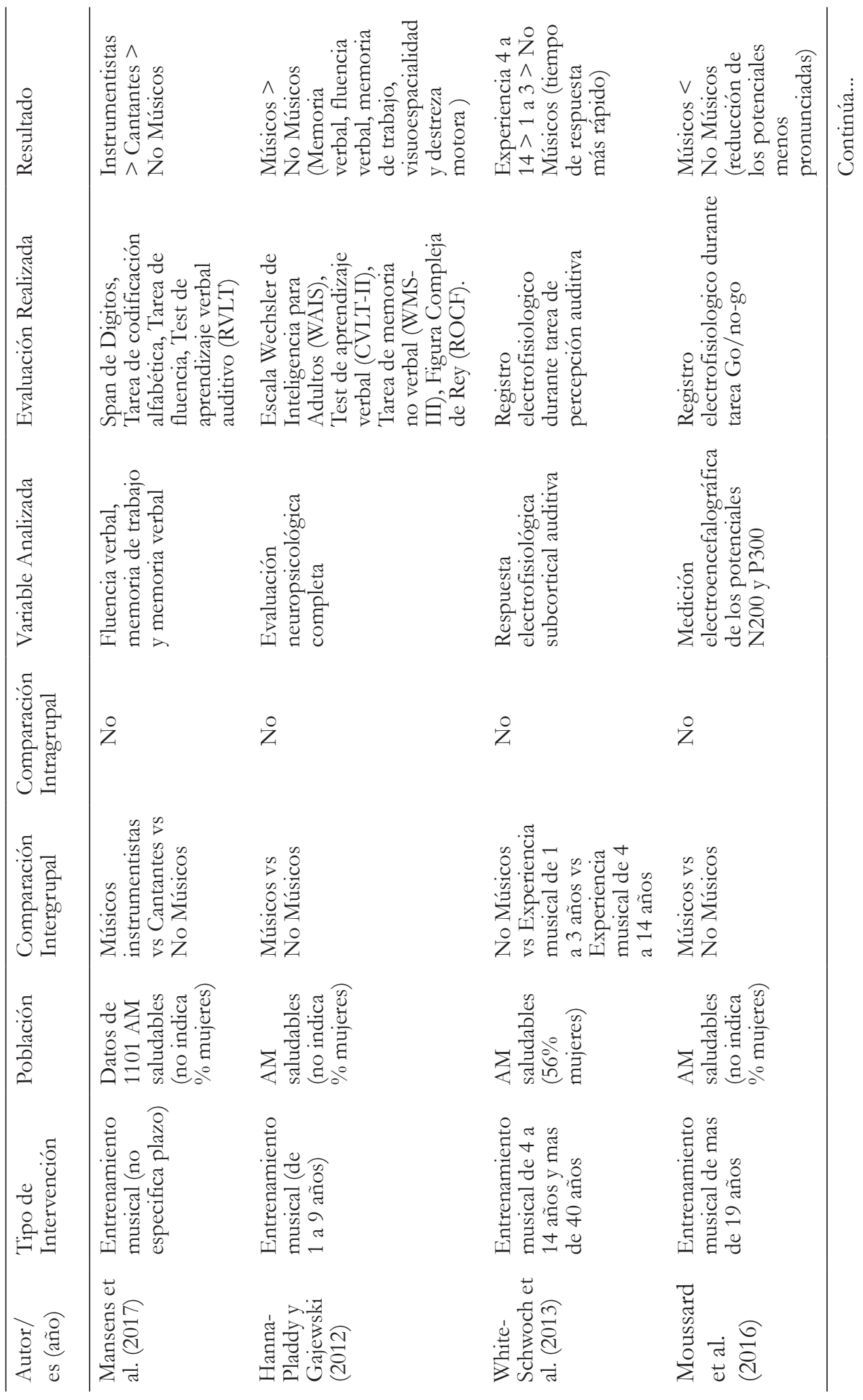

Actualidades en Psicología, 33(127), julio-diciembre 2019, 113-141 


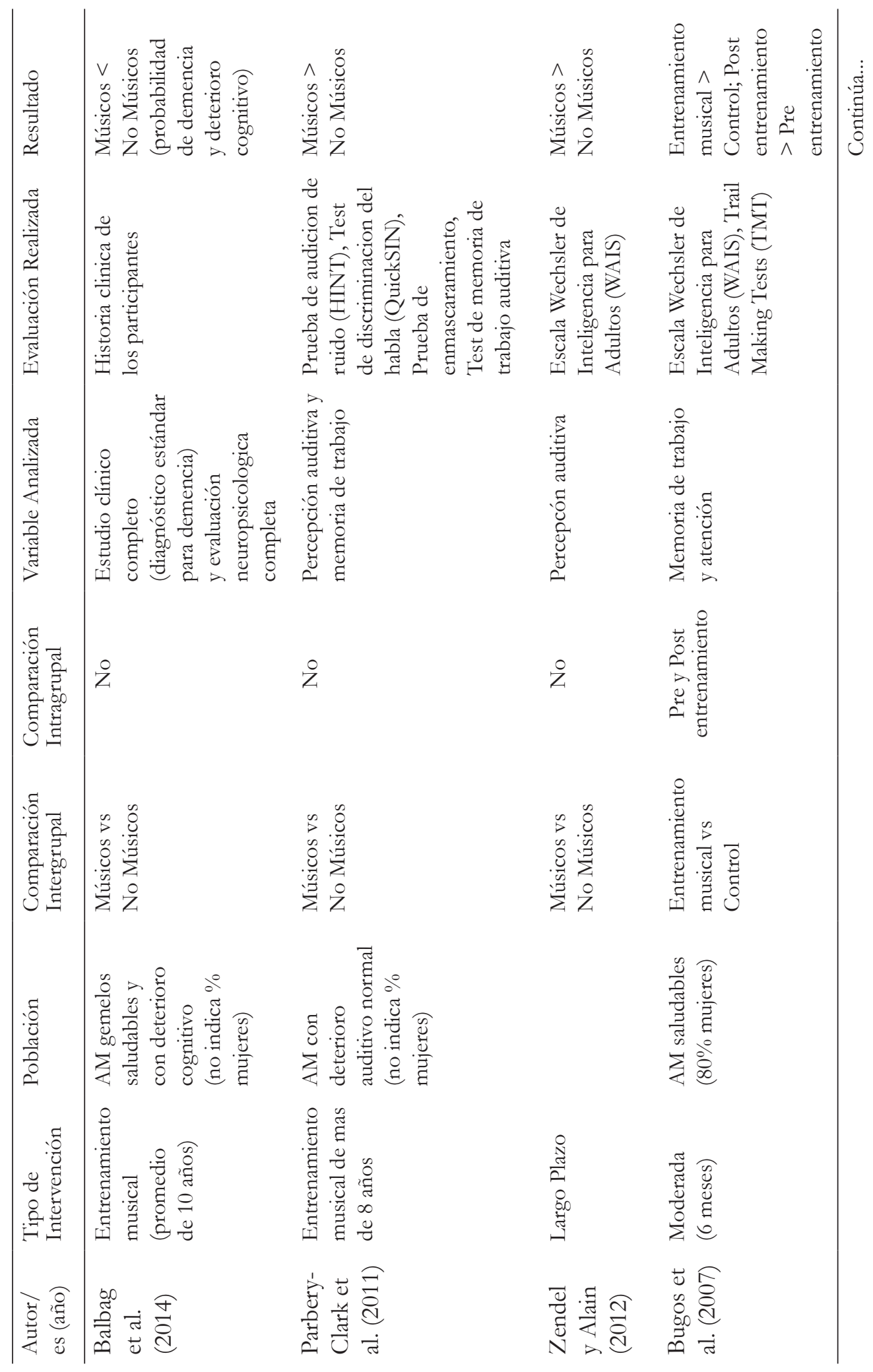

Actualidades en Psicología, 33(127), julio-diciembre 2019, 113-141 


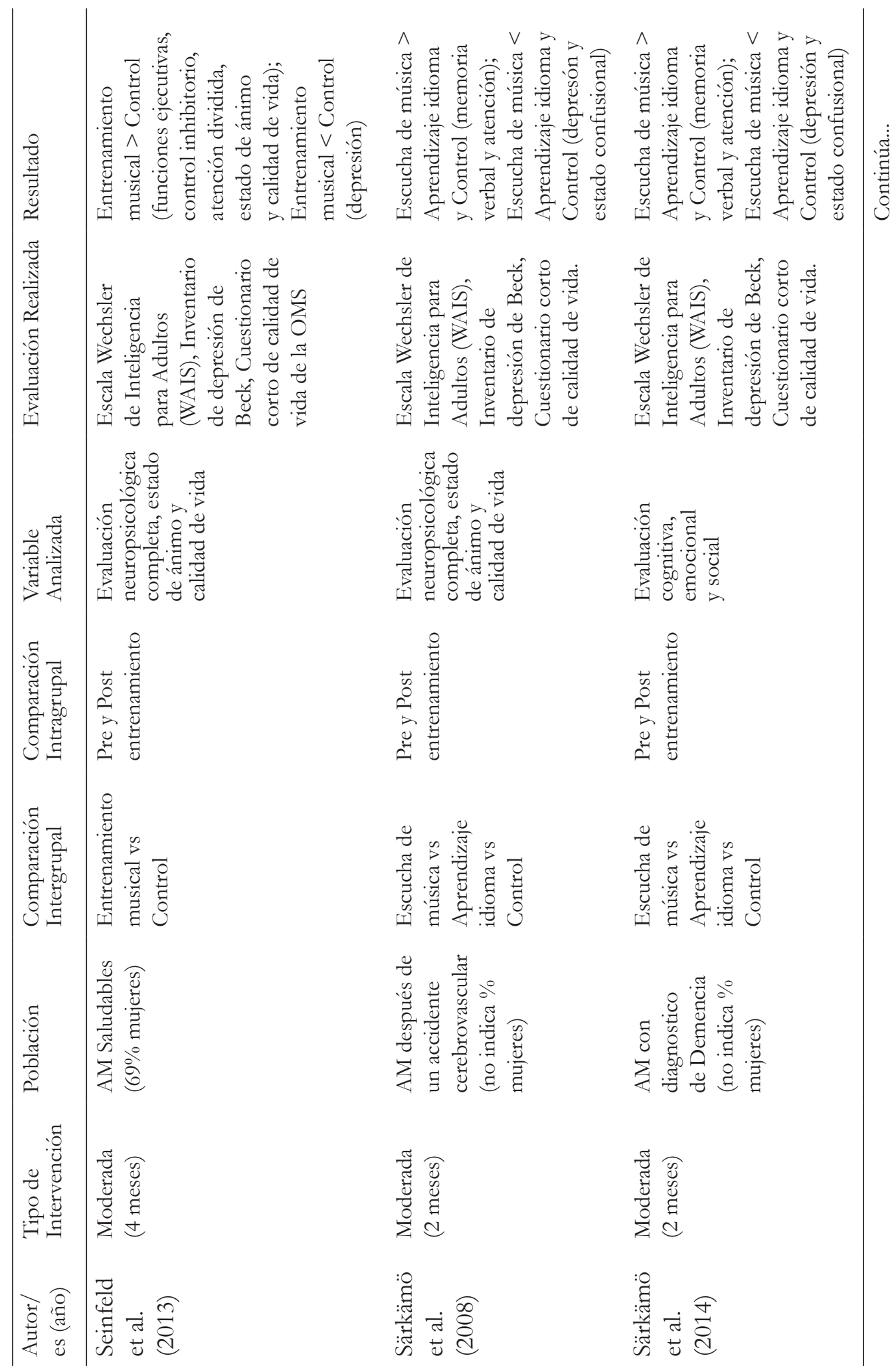

Actualidades en Psicología, 33(127), julio-diciembre 2019, 113-141 


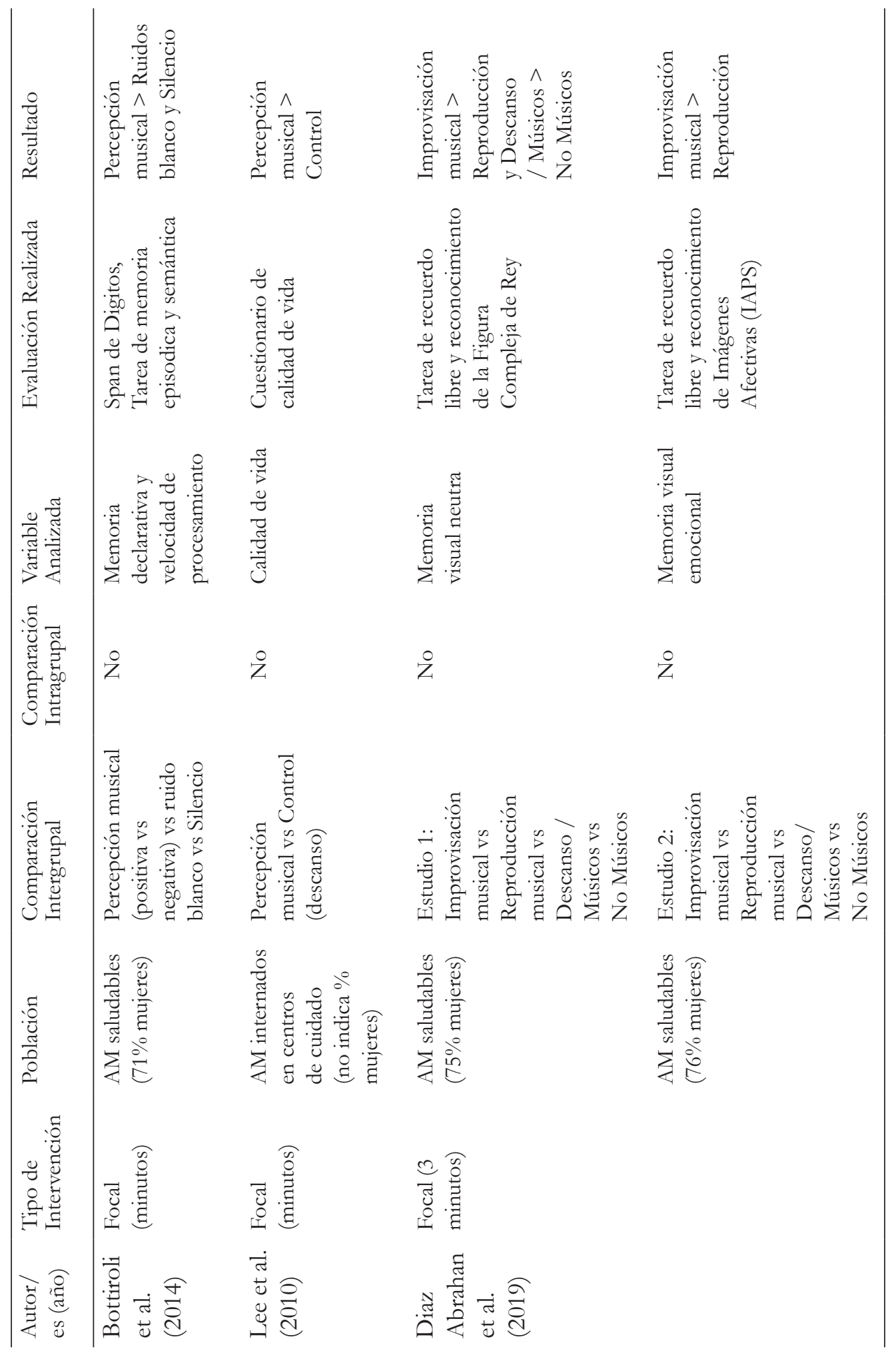




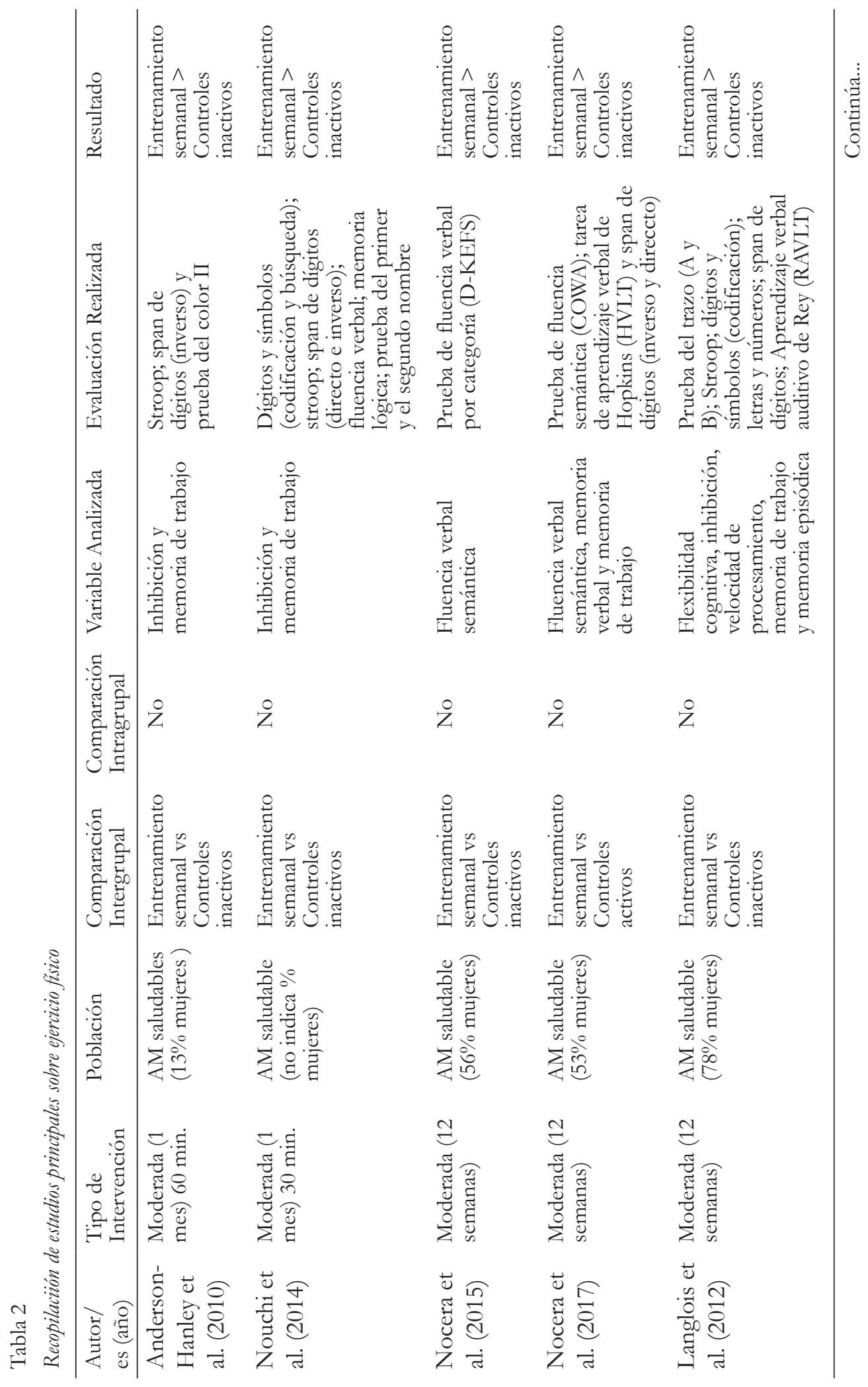

Actualidades en Psicología, 33(127), julio-diciembre 2019, 113-141 


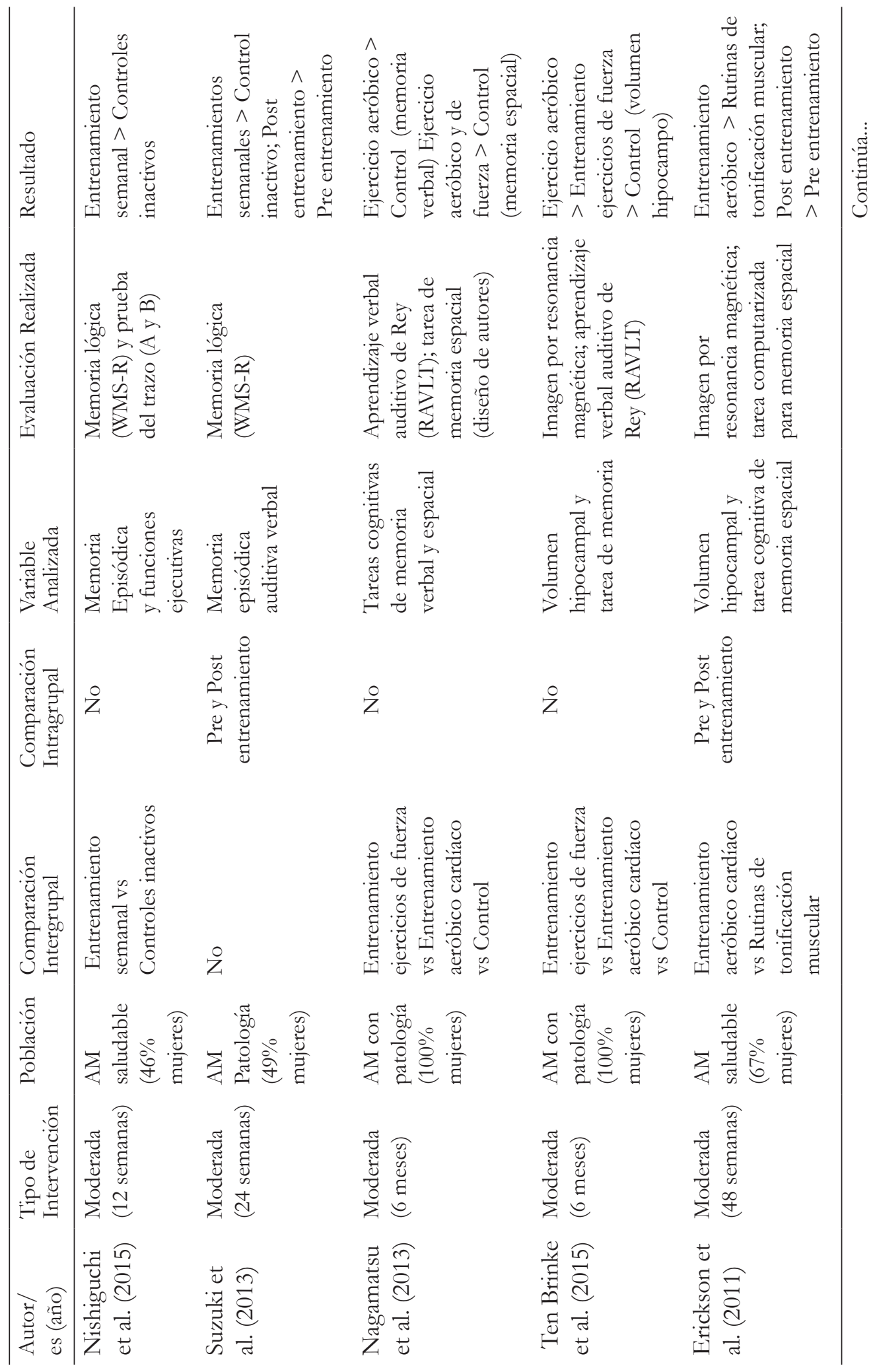

Actualidades en Psicología, 33(127), julio-diciembre 2019, 113-141 


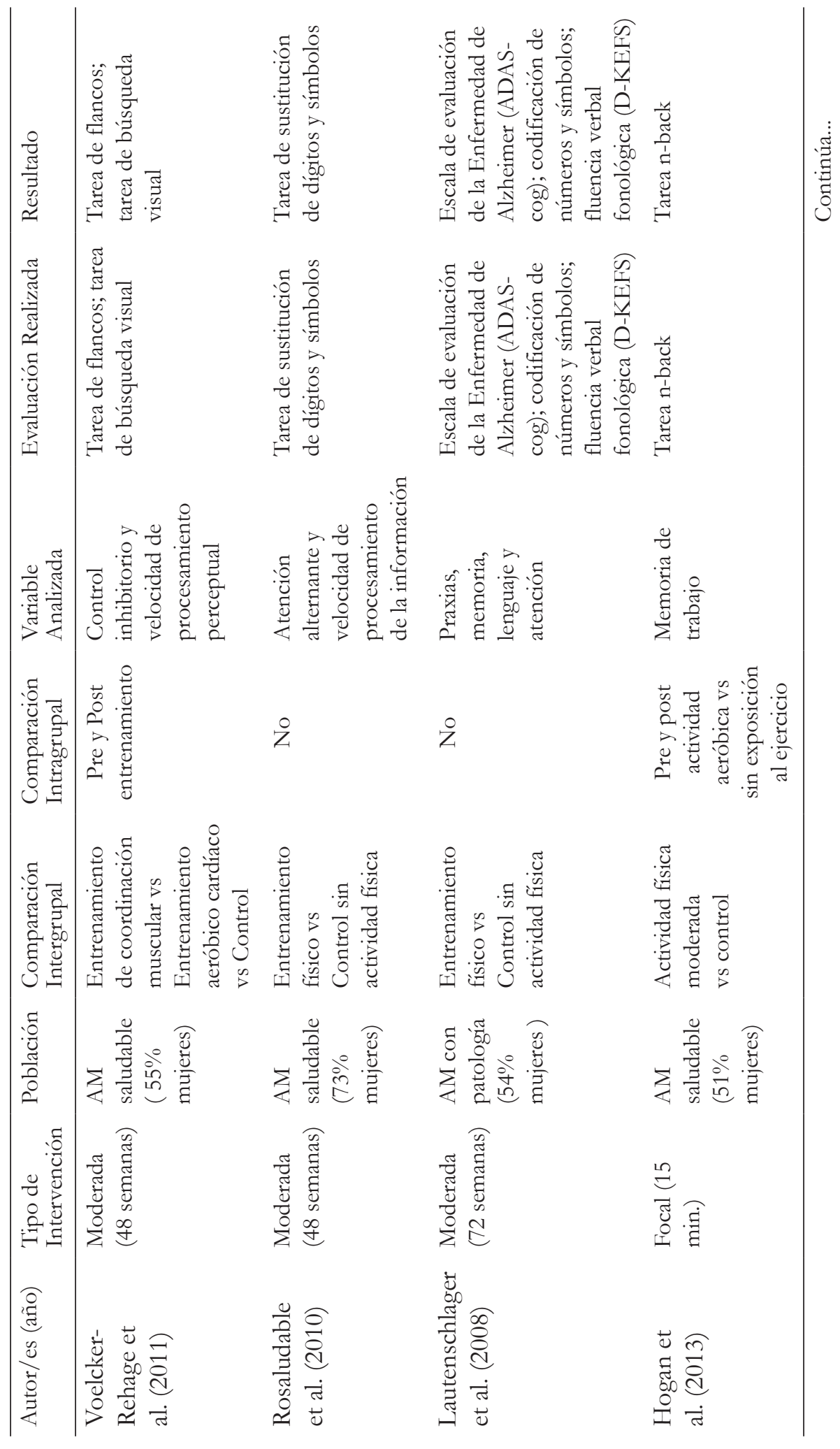

Actualidades en Psicología, 33(127), julio-diciembre 2019, 113-141 
Envejecimiento, música y actividad física 125

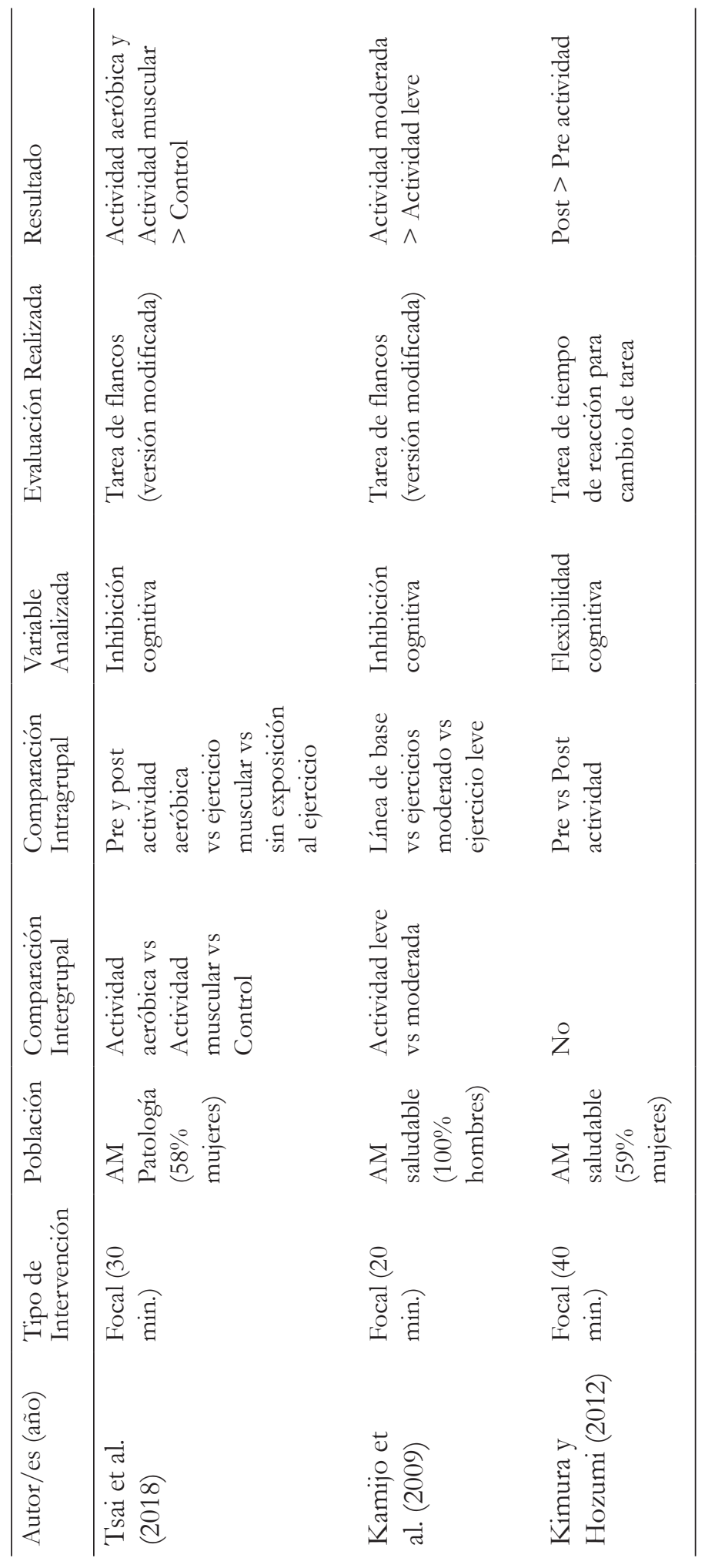

Actualidades en Psicología, 33(127), julio-diciembre 2019, 113-141 


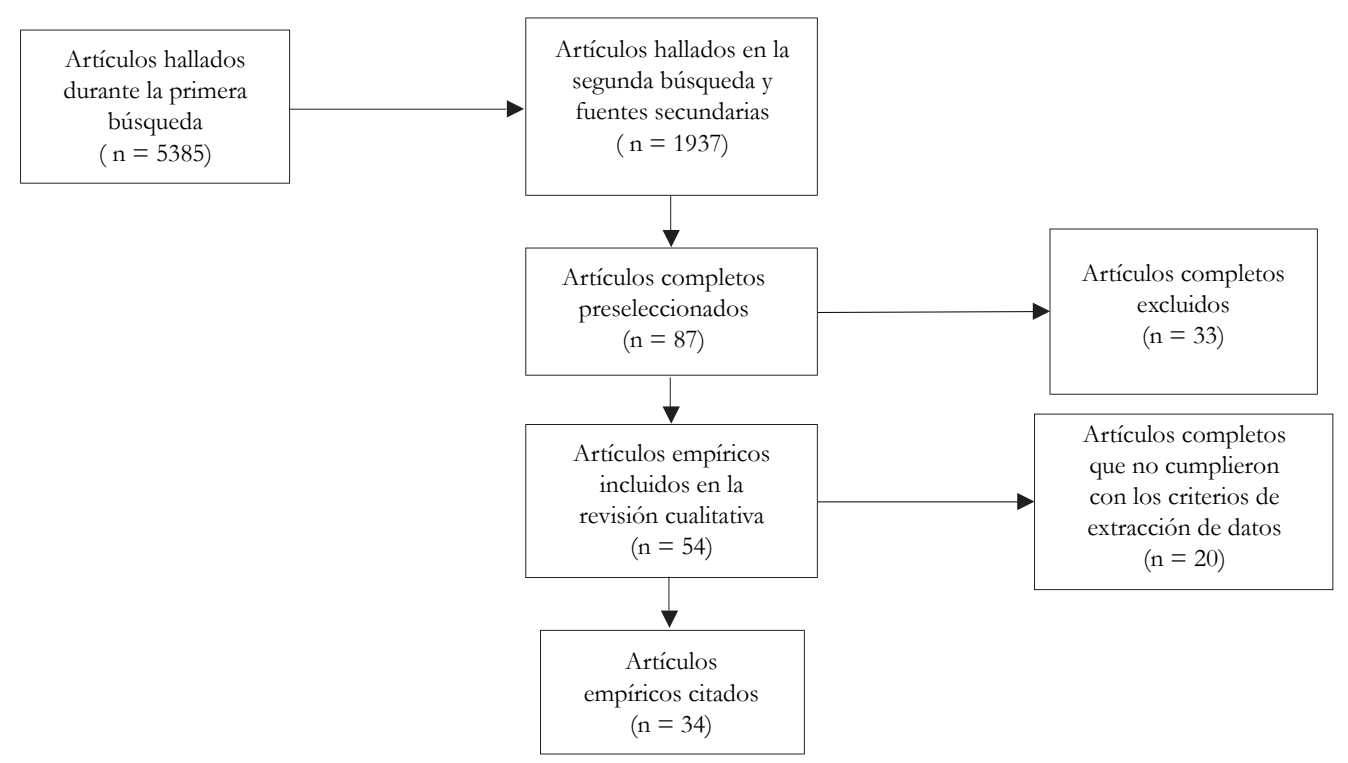

Figura 1. Flujo de selección de los artículos.

la práctica musical y el envejecimiento cognitivo mediante la comparación de individuos músicos y no músicos en dos períodos de vida diferentes (adulto joven vs. AM) y, por otra parte, la diferencia que existiría entre los músicos dependiendo del período en el cual aprendieron música. En ambos casos, evaluaron la memoria a largo plazo, la memoria auditiva y fluencia verbal, la velocidad de procesamiento y el razonamiento no verbal. En el primer estudio, los músicos presentaron un mejor rendimiento en cuanto a las mediciones de velocidad de procesamiento y la memoria auditiva verbal en comparación con los controles no músicos, sin encontrar diferencias entre los músicos jóvenes y mayores. Para la fluencia verbal, los músicos obtuvieron puntuaciones más altas que los no músicos, con un mayor beneficio para los AM. En el segundo estudio, el cual estuvo focalizado en la diferencia dependiendo del período de adquisición musical (infancia vs. edad adulta), los autores encontraron que aquellos músicos que habían comenzado a practicar durante la infancia tuvieron mejor rendimiento en fluencia fonológica que los músicos que iniciaron sus estudios durante la adultez. Por lo cual, se puede asumir que las experiencias de los individuos a lo largo de la vida influirían en la calidad del envejecimiento cognitivo.

El tipo de aprendizaje musical también puede afectar la cognición. Mansens, Deeg y Comijs (2017) compararon el rendimiento cognitivo entre AM instrumentistas musicales, 
cantantes y personas sin conocimientos musicales, evaluando diferentes medidas cognitivas. Se halló una mejora de la fluencia verbal, la memoria de trabajo y memoria verbal en los sujetos músicos al compararlos con los no músicos (Fauvel et al., 2014; Hanna-Pladdy \& MacKay, 2011). Por otra parte, encontraron diferencias dependiendo del tipo de entrenamiento musical, ya que los instrumentistas mostraron un mejor desempeño en las tareas de velocidad de procesamiento, memoria de trabajo y en el aprendizaje y retención de información verbal nueva, cuando se los comparó con los cantantes. De esta forma, se concluye que tocar un instrumento musical parecería tener más beneficios que cantar, lo que podría explicarse por la estimulación de diferentes áreas cerebrales asociadas al aspecto sensorio motor y las funciones ejecutivas puestas en juego durante el aprendizaje de un instrumento musical. Sin embargo, para esta investigación se utilizó un cuestionario auto-administrado para identificar a los músicos de la muestra, aspecto resaltado como una limitación por parte de los autores por la falta de confiabilidad de la herramienta de evaluación.

Hasta la fecha, la mayoría de los trabajos sobre la formación musical, incluso en AM, se centró en personas que tocan un instrumento continuamente a lo largo de sus vidas. Estos son casos especiales, ya que muchos adultos han incursionado con un instrumento durante la infancia, pero sin mantenerse activos en la actualidad. Una pregunta pendiente es si el entrenamiento limitado temprano en la vida deja un rastro en el sistema nervioso que envejece, afectando la función neuronal años después de que el entrenamiento se haya detenido (White-Schwoch, Woodruff, Anderson, Strait, \& Kraus, 2013). En torno a esta pregunta, se realizó un estudio que contó con la participación de AM sin deterioro cognitivo, contando con una muestra femenina del 56\%, que fueron divididos en 3 grupos dependiendo de la cantidad de años de experiencia musical que habían tenido en: ninguna ( 0 años), poca (1 a 3 años) y moderada (4 a 14 años). Se midió la respuesta electrofisiológica subcortical auditiva del tronco encefálico que se produce luego de la exposición a sonidos complejos. El grupo de entrenamiento moderado tuvo el tiempo de respuesta más rápido. Estos resultados sostienen que una mayor cantidad de entrenamiento musical temprano en la vida se asocia con una función auditiva más eficiente, décadas después de haber cesado el entrenamiento, lo que sugiere que la instrucción musical moderada generaría un cambio en el sistema nervioso central que se conservaría durante toda la vida (WhiteSchwoch et al., 2013).

Las funciones cognitivas pueden ser medidas a través de la actividad cerebral y respuestas electrofisiológicas (potenciales eléctricos evocados). En relación a ello, Moussard, Bermudez, Alain, Tays y Moreno (2016) realizaron un estudio indagando el efecto del entrenamiento musical sobre el control inhibitorio y la medición electroencefalográfica de los potenciales N200 y P300 (los cuales están asociados al control cognitivo y presentan alteraciones como producto del envejecimiento). Al comparar un grupo de músicos con uno de no músicos, los autores encontraron que las reducciones que se observan en los potenciales producto del envejecimiento son menos pronunciadas en los adultos músicos, lo que también se ve reflejado en el mejor rendimiento comportamental en una tarea de inhibición.

Un punto importante dentro de la temática del envejecimiento está determinado por las patologías asociadas a este periodo de la vida. En este punto se puede hacer mención a un 
estudio pionero, aunque preliminar, que indagaba sobre si tocar un instrumento musical proporciona protección contra la demencia al comparar la evaluación cognitiva realizada a 27 parejas de gemelos, donde uno de los hermanos presentaba antecedentes de aprendizaje musical. Los resultados de este estudio permitieron establecer una asociación significativa entre tocar un instrumento musical y la disminución de la probabilidad de aparición de demencia y deterioro cognitivo. Estos hallazgos permiten pensar a la música como un factor de prevención y protección contra la demencia y el déficit cognitivo producto de un envejecimiento patológico (Balbag et al., 2014). Sin embargo, los antecedentes en torno a esta temática son escasos y limitados, con lo cual es necesario contar con más datos para arribar a conclusiones claras.

A través de los estudios presentados se puede entrever que los músicos tienen sistemas nerviosos que responden de modo diferencial en comparación con los no músicos, debido a la plasticidad cerebral relacionada al entrenamiento. Uno de los hallazgos más prometedores de la literatura presentada es que el entrenamiento musical prolongado puede compensar las disminuciones relacionadas con la edad en las funciones neuronales y cognitivas (Hanna-Pladdy \& MacKay, 2011; Parbery-Clark, Strait, Anderson, Hittner, \& Kraus, 2011; Zendel \& Alain, 2012).

\section{Intervenciones focales y moderadas}

La mayoría de las investigaciones en los últimos años se centró en cómo el entrenamiento musical prolongado afecta al cerebro sano. Sin embargo, existen trabajos innovadores que proponen tratamientos agudos a través del uso de actividades de estimulación, las cuales están definidas como la intervención en un momento particular (Pontifex, Hillman, Fernhall, Thompson, \& Valentini, 2015). Vinculadas a la música y orientadas a personas con o sin conocimientos musicales, estas propuestas incluyen tocar un instrumento musical, escuchar o crear música, entre otras, las cuales estimulan una gran variedad de funciones cognitivas, con la capacidad de inducir plasticidad cerebral y compensar el declive cognitivo que conlleva el envejecimiento (Zatorre \& McGill, 2005).

Bugos, Perlstein, McCrae, Brophy y Bedenbaugh (2007) presentan al entrenamiento musical como una intervención cognitiva óptima que integra varias redes neuronales, las cuales permiten prevenir, así como también rehabilitar, el declive cognitivo producto de la edad. Los autores realizaron un estudio mediante el cual brindaron clases individuales de piano y teoría musical, durante 6 meses, a AM sin conocimientos en música. Contaban, además, con un grupo control que no recibió entrenamiento musical, con el objetivo de prevenir el declive cognitivo patológico. Se evaluó la memoria de trabajo y atención, antes y después del entrenamiento, y se encontró un mejor rendimiento por parte del grupo con la intervención musical en comparación con el grupo control. A su vez, se hallaron diferencias intragrupales, ya que el grupo musical logró mejorar su rendimiento en relación con la línea base establecida, aumento que no se observó en el grupo control. Teniendo en cuenta el grado de deterioro cognitivo que acompaña al envejecimiento, el aprendizaje musical puede servir como una técnica útil para mejorar las funciones cognitivas de los AM.

Varios estudios sugieren que las experiencias musicales relativamente breves, de menos de 6 meses, pueden mejorar varios aspectos de la salud física y cognitiva de los adultos 
mayores. Cuatro meses de entrenamiento musical, a través de clases de piano, mostraron tener un impacto positivo sobre las funciones cognitivas de los participantes, en el estudio realizado por Seinfeld et al. (2013). En este estudio, el rendimiento cognitivo de los AM que aprendieron música fue comparado con un grupo control de sujetos que realizaba otro tipo de actividad de la vida diaria (ejercicio físico, clases de computación o de pintura); los autores encontraron que las mediciones de las funciones ejecutivas, el control inhibitorio y la atención dividida mejoraron en las evaluaciones post entrenamiento musical, incremento que no se observó en el grupo control. Por otra parte, las mejoras como producto del entrenamiento musical no solo se observaron en la evaluación de las funciones cognitivas, ya que los autores también reportaron una disminución de la presencia de síntomas de depresión, aumento del estado de ánimo y de los indicadores de calidad de vida. Aunque los resultados muestran las potencialidades de las propuestas musicales, los autores identifican el tamaño de la muestra con la que contaron $(N=30)$ como una limitación del estudio, lo que imposibilita la generalización de resultados hacia otras poblaciones de adultos mayores.

Los estudios también se han centrado en AM con déficit o patología neurocognitiva. Por ejemplo, se ha demostrado que la escucha de música diariamente, a lo largo de dos meses, ayuda a la recuperación cognitiva y la mejora del estado emocional en AM después de un accidente cerebrovascular (Särkämö et al., 2008); mejora la dinámica de la marcha y reduce el riesgo de caídas (Trombetti et al., 2011). En particular, un estudio de Särkämö et al. (2014) evaluó varios aspectos de la salud física y cognitiva de AM con demencia que completaron ya sea 10 semanas de entrenamiento vocal, 10 semanas de escucha musical o quienes no recibieron intervención musical. Ambos grupos de intervenciones musicales mostraron un estado de ánimo mejorado, mejor rendimiento en tareas de memoria episódica remota, atención y funciones ejecutivas, en comparación al grupo control. Sin embargo, una limitación importante destacada por los mismos autores es la generalización de la muestra, ya que no se focalizaron en un tipo particular de cuadro demencial, lo que condiciona las conclusiones específicas que pueden extraerse sobre la efectividad de la intervención musical.

El uso de la música de fondo es una propuesta que se caracteriza por la escucha de música mientras el sujeto realiza una tarea (Radocy \& Boyle, 1988) y que ha mostrado resultados positivos en cuanto a la mejora en diversas funciones cognitivas (Angel, Polzella, \& Elvers, 2010; Ferreri, Aucouturier, Muthalib, Bigand, \& Bugaiska, 2013; Kang \& Williamson, 2013). Aunque es un tratamiento con potencialidades, la mayor parte de estas investigaciones se realizaron con sujetos jóvenes. Con el fin de brindar evidencia en personas de la tercera edad, se llevó a cabo un estudio (Bottiroli, Rosi, Russo, Vecchi, \& Cavallini, 2014) donde evaluaron la memoria declarativa y la velocidad de procesamiento al mismo tiempo que los AM, sin conocimientos musicales, escuchaban uno de tres estímulos sonoros (una pieza musical con características emocionales positivas, una pieza musical negativa o ruido blanco) o permanecían en silencio. Los resultados de este estudio indicaron que los grupos que escucharon música positiva presentaron un mejor rendimiento en su velocidad de procesamiento, mientras que la memoria, tanto episódica como semántica, se vio beneficiada por ambos tipos de piezas musicales. 
La calidad de vida también se evaluó en un estudio donde los AM estaban divididos en un grupo expuesto a una intervención musical de 30 minutos repetida a lo largo de cuatro semanas y otro grupo que permaneció descansando. Se halló una mejora en la calidad de vida en el grupo de música en comparación con los controles (Lee, Chan, \& Mok, 2010).

Dentro del abanico de posibilidades en cuanto a las actividades musicales se encuentran aquellas que implican la producción musical, ya sea reproduciendo patrones preestablecidos o creando contenidos musicales novedosos (Díaz Abrahan, Shifres, \& Justel, 2019). Este enfoque fue tomado en una investigación que utilizó la improvisación musical como una intervención focal para modular la memoria de AM saludables. En este estudio, los participantes realizaron una tarea de aprendizaje de información de tipo visual de contenido neutral (estudio 1) o emocional (estudio 2) y, posteriormente, fueron expuestos a una de tres actividades, con una duración de 3 minutos: una improvisación musical (los participantes realizaban una creación musical grupal), una reproducción musical (los participantes imitaban grupalmente un patrón rítmico) o la permanencia en descanso (los participantes se mantenían grupalmente en silencio).

Luego de evaluar la memoria del contenido aprendido, los autores hallaron una mejora en el rendimiento mnémico por parte de los AM que realizaron una improvisación musical luego de la adquisición de información, lo que indicaría que dentro de las propuestas musicales existe un efecto específico relacionado con la creatividad musical. Por otra parte, este mismo estudio incluyó una segunda variable independiente definida por los antecedentes musicales de los participantes, dividiendo a la muestra entre músicos y no músicos. Se encontró que los AM con más de 5 años de entrenamiento musical presentaban un mejor rendimiento de la memoria de tipo visual neutra que los no músicos (Díaz Abrahan, Shifres, \& Justel, 2019). Empero, los investigadores destacan dos limitaciones importantes vinculadas a la muestra: por un lado, el desbalance por género, puesto que la mayor parte de los AM eran mujeres; y, por otro lado, la necesidad de contar con datos específicos que permitan establecer diferencias entre el grupo de los músicos; por ejemplo, si actualmente se desempeñan musicalmente o la edad de inicio del aprendizaje musical, ya que estos aspectos no fueron contemplados en el estudio y podrían establecer diferencias en el rendimiento cognitivo de los participantes.

De esta forma, se puede concluir que las actividades musicales permiten paliar el declive cognitivo, no solo en un envejecimiento normal, sino también abordar el déficit en el envejecimiento patológico. Sin embargo, se debe destacar que las investigaciones en torno a las propuestas de corto plazo o focales son escasas, lo que permite identificar una laguna en la literatura que incentiva a pensar investigaciones sobre la implementación de intervenciones agudas para el abordaje de las funciones cognitivas que presentan declive, producto del envejecimiento.

Actividad fisica y adultos mayores

A continuación se desarrollarán las intervenciones aeróbicas-físicas, tanto a largo como corto plazo, que beneficiarían el funcionamiento cognitivo de AM. 


\section{Intervenciones moderadas}

A lo largo de los subsiguientes párrafos, se abordarán estudios donde los entrenamientos se repiten periódicamente, con una frecuencia semanal de entre 1 y 3 encuentros. Bajo esta temática, existen trabajos que utilizan planificaciones que no superan el mes de duración y comparan grupos controles inactivos con grupos que reciben entrenamientos semanales. Incluso, con estos períodos breves, se halló que los AM de los grupos activos mejoraban significativamente su rendimiento en tareas de inhibición y memoria de trabajo (Anderson-Hanley, Nimon, \& Westen, 2010), así como velocidad de procesamiento y memoria episódica, inhibición y fluencia verbal (Nouchi et al., 2014) en comparación con los grupos inactivos. Sin embargo, estas investigaciones reportan la necesidad de estudiar más profundamente la naturaleza y efectividad de los tratamientos empleados.

Excediendo las 4 semanas de intervención, una investigación registró el desempeño cognitivo de AM que realizaban un entrenamiento aeróbico durante 12 semanas y lo comparó con un grupo control sin actividad. Como resultado, los investigadores hallaron que el grupo aeróbico se desempeñaba significativamente mejor que el grupo control en tareas de fluencia verbal (Nocera, McGregor, Hass, \& Crosson, 2015). Adicionalmente, otro estudio llevado a cabo por los mismos autores reveló que dicha diferencia, favorable a la actividad aeróbica, se sostenía aun modificando la condición control: si bien atravesaban tratamientos que los activaban físicamente, estos no implicaban una exigencia cardiorrespiratoria destacable (Nocera, Crosson, Mammino, \& McGregor, 2017). A su vez, tal metodología fue efectiva en la modulación de la flexibilidad cognitiva, inhibición, velocidad de procesamiento y memoria de trabajo (Langlois et al., 2012), así como memoria episódica y flexibilidad (Nishiguchi et al., 2015). Si bien estos programas obtuvieron resultados favorables, la no implementación de un grupo control activo podría explicar los beneficios con base en el contacto social recibido por el grupo experimental. Este aspecto, así como el tamaño relativamente pequeño de las muestra, resultan limitaciones sensibles destacadas por los autores de estos trabajos.

Ampliando el tiempo de duración de las intervenciones, un estudio comparó las funciones cognitivas de los sujetos antes y después de 24 semanas de inactividad (grupo control), o bien, luego de un entrenamiento combinado de fuerza, ejercicio aeróbico y cognitivo. Hallaron que, al finalizar el entrenamiento, los participantes físicamente activos mejoraron su desempeño en tareas de memoria episódica auditiva verbal, tanto inmediata como diferida. Este efecto no se observó en el grupo control. Además, los resultados de este grupo demostraron ser significativamente peores que los registrados en su propia línea de base (Suzuki et al., 2013). En relación con el tema, pueden citarse dos estudios que contaron con una muestra de AM únicamente de género femenino. En ambos se aplicaron 3 tipos de tratamiento a lo largo de 6 meses: grupo control, entrenamiento con ejercicios de fuerza, y entrenamiento aeróbico cardíaco. Se halló que, mientras ambos grupos de actividad física obtenían tiempos de respuesta más cortos en una prueba de memoria espacial, solo aquellos que participaron del ejercicio aeróbico mejoraron su desempeño en una tarea de memoria verbal con interferencia (Nagamatsu et al., 2013). El segundo estudio mencionado trabajó solo con la prueba de memoria verbal y obtuvo los mismos resultados. Se encontró, además, una asociación entre dicha variable y el volumen del 
hipocampo que presentaban los sujetos, el cual era mayor solo en el grupo de ejercicio aeróbico (Ten Brinke et al., 2015). Sin embargo, los autores son cautelosos con dichos resultados, estableciendo la necesidad de explorar más profundamente la relación entre las variables mencionadas. Por último, en todos los casos se menciona la necesidad de emplear muestras más grandes.

Con una intervención de 48 semanas, una investigación empleó la actividad física aeróbica como grupo experimental mientras que el grupo control siguió rutinas de tonificación muscular. La comparación de evaluaciones previas y posteriores, obtenidas mediante resonancia magnética, demostró un aumento significativo del volumen en hipocampo para el grupo aeróbico y una disminución del mismo en aquellos que conformaron el grupo de tonificación muscular. A su vez, encontraron que los sujetos con niveles cardiorrespiratorios más altos, obtuvieron mejores resultados al evaluar su memoria espacial (Erickson et al., 2011). Además de estos resultados favorables para la memoria, diferentes estudios que han empleado programas de larga duración (48 semanas de entrenamiento o superiores) dan cuenta de beneficios en el control inhibitorio y velocidad de procesamiento perceptual (Voelcker-Rehage, Godde, \& Staudinger 2011), atención alternante y velocidad de procesamiento de la información (Rosano et al., 2010), praxias, memoria y lenguaje (Lautenschlager et al., 2008). Como se mencionó previamente, el tamaño de la muestra representa una limitación.

\section{Intervenciones focales}

En el presente apartado se tratarán aquellas investigaciones que emplean dosis agudas de ejercicio. Estas son entendidas como aquellas que no superan la hora de duración y que ocurren por una única vez en un lugar y momento determinados.

En torno a la temática, se comparó el desempeño en la memoria de trabajo de los sujetos antes y después de una actividad aeróbica o sin exposición al ejercicio. Los resultados indicaron que, en el grupo experimental, los tiempos de respuesta disminuían significativamente de modo posterior al ejercicio, mostrando un mejor desempeño cognitivo. Este efecto no se observó en el grupo control (Hogan et al., 2013). En línea con estos resultados, un trabajo empleó una metodología similar, pero adicionando un grupo de ejercicio no aeróbico de fuerza muscular como tercer tratamiento y evaluando la inhibición cognitiva. Se halló un mejor desempeño en ambos grupos activos en comparación al grupo control (Tsai, Ukropec, Ukropcová, \& Pai, 2018). A pesar de los efectos favorables observados mediante las dosis agudas, los autores proponen estudios complementarios, adicionando entrenamientos repetitivos y sostenidos en el tiempo como variable comparativa.

En línea con los resultados mencionados, un estudio evaluó la inhibición cognitiva, al comparar grupos experimentales que se diferenciaban en cuanto al nivel de intensidad de la ejercitación. Al cotejar con los resultados obtenidos en la línea de base, se halló que los desempeños mejoraban significativamente luego de realizar ejercicios a intensidad moderada, sin que la exigencia leve afectara los mismos (Kamijo et al., 2009). En relación con la investigación precedente, un estudio comparó dos programas de baile de salón donde diferencialmente se realizaban movimientos estereotipados y repetitivos, o bien series de movimientos que implicaban combinaciones en constante variación (lo cual 
implica mayor demanda cognitiva para los sujetos). Se evaluó la flexibilidad cognitiva, antes y después de la actividad, y se encontraron mejoras en ambos grupos; sin embargo, los participantes del grupo con mayor demanda cognitiva tuvieron un mejor desempeño que sus compañeros con baja demanda (Kimura \& Hozumi, 2012).

A pesar de que las intervenciones focales han arrojado resultados favorables, las investigaciones que adoptan este tipo de metodología son escasas al compararlas con las de largo plazo. Esto puede deberse al hecho que los tratamientos focales requerirían de un nivel de intensidad entre moderado y vigoroso (Borg, 1970) para hallar resultados favorables. Debido a la exigencia que dichas prácticas representan a nivel corporal, y a fin de salvaguardar la salud de los individuos, las investigaciones que cuentan con la participación de AM realizan una selección exhaustiva y minuciosa de la muestra (Kimura \& Hozumi, 2012; Tsai et al., 2018). Por ello, hallar una muestra representativa que al mismo tiempo cumpla con todos los requisitos en cuanto a la salubridad esperada, puede resultar una tarea ardua. Por otro lado, si además se considera que en dicha población los efectos de los tratamientos se observan a mediano y largo plazo (Erickson et al., 2011; Rosano et al., 2010; Voelcker-Rehage et al., 2011), podría entenderse que los investigadores se vean inclinados a optar por intervenciones comprendidas en semanas o meses, creando así programas de entrenamiento a mediano y largo plazo.

\section{Discusión}

El proceso de envejecimiento es un fenómeno universal que se asocia a una declinación general de las funciones fisiológicas, conductuales y cognitivas impactando en la calidad de vida de los AM (Díaz \& Pereiro, 2018). La proporción de personas mayores de 65 años está aumentando más rápidamente que cualquier otro grupo etario en casi todos los países (OMS, 2017) y se estima que para el año 2050 habrá 114 millones de personas con demencia, siendo una de las principales causas de discapacidad y dependencia en la población de AM (Iuliano et al., 2015). Con ello, la búsqueda de estrategias que no solo promuevan el envejecimiento saludable, sino también que prevengan los procesos patológicos, es una temática de capital interés para la sociedad, aspecto que guio la presente revisión sistemática.

El objetivo del artículo fue presentar y analizar las últimas investigaciones en torno a propuestas musicales y físicas, en pos de lograr un envejecimiento saludable y enfrentar el declive cognitivo. Esta temática viene sumando evidencias positivas e incrementado los conocimientos y las intervenciones tendientes a abordar los cambios que se producen en las funciones cognitivas de los individuos conforme avanza la edad (Román Lapuente \& Sánchez Navarro, 1998).

Durante el envejecimiento, las principales dificultades cognitivas están asociadas a los procesos de orden superior (Díaz \& Pereiro, 2018), los cuales se encuentran especialmente entrenados y estimulados con las actividades musicales y el ejercicio físico. Por lo tanto, hay una buena razón para especular que estos dos factores ambientales podrían tener una influencia positiva en la cognición, para este período de la vida. De hecho, los estudios que se centran en esta temática y el efecto en la cognición en sujetos de edad avanzada tienden a confirmar esta suposición (Bugos et al., 2007; Erickson et al., 
2011; Hanna-Pladdy \& MacKay, 2011), al encontrar rendimientos mejorados en diversas medidas cognitivas (Bugos et al., 2007; Fauvel et al., 2014; Mansens et al., 2017; Rosano et al., 2010; Särkämö et al., 2014; Särkämö, Altenmüller, Rodríguez-Fornells, \& Peretz, 2016).

El estilo de vida de los AM, ya sea asociado a la música o a la actividad deportiva, repercute en el desarrollo cognitivo durante el envejecimiento. En este sentido, el entrenamiento musical puede compensar las disminuciones, producto del envejecimiento normal, en la memoria, la velocidad del procesamiento y las funciones ejecutivas (Hanna-Pladdy \& MacKay, 2011; Parbery-Clark et al., 2011; Zendel \& Alain, 2012). Sin embargo, determinar si una persona es músico o presenta una experiencia musical destacable, parece ser un aspecto metodológico que requiere atención, lo cual representa una limitación reportada también por parte de los mismos investigadores de los estudios analizados, quienes indican la falta de confiabilidad de los cuestionarios auto administrados que determinan el tipo y el tiempo de la experiencia musical de los participantes. Resulta necesario contar con herramientas específicas que evalúen detalladamente los diferentes aspectos que involucran el quehacer musical, con el fin de caracterizar adecuadamente la muestra de estudio e identificar específicamente el efecto de los entrenamientos ambientales.

Por otra parte, y en cuanto a la actividad física como actividad de la vida diaria, se ha visto que el desempeño en memoria de trabajo, flexibilidad cognitiva y memoria episódica es significativamente mejor en aquellos AM que poseen una capacidad cardiorrespiratoria superior producto del entrenamiento físico (Hayes et al., 2014).

Ambas propuestas, música y actividad física, presentan cierta flexibilidad que les permite ser implementadas en la población en general (es decir, sin la necesidad de ser músicos profesionales o deportistas de alto rendimiento), haciendo uso de los recursos que las personas disponen al momento de la actividad (Bottiroli et al., 2014; Lee et al., 2010). En este punto, tanto las actividades musicales como las aeróbicas, implementadas como entrenamientos de corto tiempo (semanas o meses), han mostrado resultados positivos en lo que respecta a la memoria de trabajo, la velocidad del procesamiento, la inhibición cognitiva, los estados de ánimo, la atención, fluencia verbal y flexibilidad cognitiva. Un punto favorable en las investigaciones, especialmente en los estudios de cohorte, es la incorporación de grupos control. Aunque con potencialidades, los estudios de esta naturaleza son escasos, lo que se traduce en la necesidad de contar con más evidencia para arribar a conclusiones sostenidas.

Finalmente, se encuentran las propuestas de tipo focal, las cuales se caracterizan por la intervención implementada una única vez y/o en un momento determinado de un desempeño cognitivo. En este sentido, el uso de la música de fondo en simultáneo a la realización de una tarea cognitiva ha mostrado ser beneficiosa en tareas de memoria declarativa, velocidad de procesamiento y la calidad de vida de los AM (Bottiroli et al., 2014; Lee et al., 2010).

En cuanto a las intervenciones aeróbicas agudas, los estudios mostraron mejoras en la memoria de trabajo y la inhibición cognitiva (Hogan et al., 2013; Kamijo et al., 2009; Tsai et al., 2018). A pesar de que estos trabajos focales han arrojado resultados favorables, las investigaciones que adoptan este tipo de metodología no abundan en la literatura, un hueco que se ha podido observar tanto para las investigaciones sobre música como las de actividad física. 
Hay aspectos a destacar en pos de mejorar los estudios y brindar especificidad a las actividades. En este sentido, ambas intervenciones presentan un gran abanico de posibilidades en cuanto a las propuestas que pueden utilizarse pensando en el desempeño cognitivo. El aprendizaje de un instrumento musical y la escucha de música durante el desarrollo de una tarea cognitiva fueron las propuestas que se destacaron en la mayor parte de los estudios; sin embargo, la música brinda muchas posibilidades que quedan por fuera de estas investigaciones, como por ejemplo la composición e improvisación musical, entre otras (Abrahan \& Justel, 2015). Si bien la actividad aeróbica requiere una selección específica de la muestra dependiendo de las condiciones físicas de los AM, existen diferentes y diversas propuestas con el objetivo de mejorar la calidad de vida de las personas de la tercera edad.

Por otra parte, otro punto débil en cuanto a los aspectos metodológicos de las investigaciones presentadas en este artículo, especialmente las dedicadas la implementación de intervenciones moderadas y focales, es la ausencia de grupos activos como condiciones controles. Tanto en los antecedentes musicales, y en menor medida en los deportivos, las mayores comparaciones de los grupos experimentales es con controles inactivos. En este punto, los resultados podrían explicarse por la mera actividad de los ancianos o la interacción social entre los participantes, lo que le quita fuerza a los resultados obtenidos sobre la efectividad de los tratamientos.

Otro punto que merece atención es la población que participa de las investigaciones, es decir el desbalance que hay por género en los estudios de adultos mayores, pues es mayor el porcentaje de mujeres participantes, con una mayor incidencia en los estudios dedicados a la implementación de una propuesta musical. En este punto, existe una tendencia a encontrar más participación en propuestas sociales por parte de las mujeres adultas mayores, lo cual sería la causa del desbalance en los estudios reportados (Hanna-Pladdy \& MacKay, 2011). Este aspecto que debería contemplarse como una variable interviniente en los posibles futuros estudios.

Si bien existen resultados cognitivos compartidos, se puede observar que tanto la música como la actividad física proporcionan efectos particulares asociados a las características propias de cada actividad. En este sentido, pensar y poner en evidencia programas que combinen particularidades de ambas propuestas parece ser un camino con grandes potencialidades. Resulta de suma importancia emprender investigaciones que permitan establecer el efecto de la estimulación ambiental musical y física, ya sea través del entrenamiento a largo plazo como con intervenciones agudas, para favorecer las reservas cerebral y cognitiva, con el propósito final de mejorar la actuación de estas personas en la vida cotidiana, posibilitar un desempeño adaptado al mundo en el que viven y asegurar una participación social efectiva de los AM.

Finalmente, esta revisión sistemática presenta ciertas limitaciones. Por una parte, la búsqueda bibliográfica se realizó en las siguientes bases de datos: Redalyc, Scielo, Science Direct, PubMed, y Taylor \& Francis. Sin embargo, esta lista podría ampliarse para futuros estudios. Por otra parte, podría especificarse la búsqueda utilizando, en particular, variables cognitivas como palabras clave, elemento que no fue considerado en el presente procedimiento. Estos aspectos serán contemplados en futuros estudios de revisión. 
En conclusión, las posibilidades en torno a ambos factores ambientales brindan no solo opciones ecológicas, simples y accesibles a la comunidad (Bugos et al., 2007), sino que también proveen propuestas motivadoras para las personas, preservando los objetivos de prevención y rehabilitación cognitiva ante el declive producto del envejecimiento y el cuidado general de la salud. En este sentido, la música y la actividad física proveen opciones que pueden ser aprovechadas teniendo en cuenta el compromiso y los beneficios cognitivos que se reportaron a lo largo del presente artículo, y a su vez haciendo provecho del plus motivacional que brindan, al ser actividades de gran disfrute por parte de la población en general, lo que sin dudas brinda una gran adherencia a los tratamientos clínicos y los programas de prevención y promoción de la salud para la tercera edad.

\section{Referencias}

Abrahan, V. \& Justel, N. (2015). La improvisación musical. Una mirada compartida entre la musicoterapia y las neurociencias. Psicogente, 18(34), 372-384. doi: 10.17081/ psico.18.34.512

Adam, S., Bonsang, E., Grotz, C., \& Perelman, S. (2013). Occupational activity and cognitive reserve: Implications in terms of prevention of cognitive aging and Alzheimer's disease. Clinical Interventions in Aging, 8, 377-390 doi: 10.2147/CIA.S39921

Anderson-Hanley, C., Nimon, J. P., \& Westen, S. C. (2010). Cognitive health benefits of strengthening exercise for community-dwelling older adults. Journal of Clinical and Experimental Neuropsychology, 32(9), 996-1001. doi: 10.1080/13803391003662702

Angel, L. A., Polzella, D. J., \& Elvers, G. C. (2010). Background music and cognitive performance. Perception Motor Skills, 11(3 Pt 2), 1059-1064 doi: 10.2466/pms.110.3c.1059-1064

Balbag, M. A., Pedersen, N. L., \& Gatz, M. (2014). Playing a musical instrument as a protective factor against dementia and cognitive impairment: A population-based twin study. International Journal of Alzheimer's Disease, 2014, 836748. doi: 10.1155/2014/836748

Borg, G. (1970). Perceived exertion as an indicator of somatic stress. Scandinavian Journal of Rehabilitation Medicine, 2(2), 92-98.

Bottiroli, S., Rosi, A., Russo, R., Vecchi, T., \& Cavallini, E. (2014). The cognitive effects of listening to background music on older adults: Processing speed improves with upbeat music, while memory seems to benefit from both upbeat and downbeat music. Frontiers in Aging Neuroscience, 6, 284. doi: 10.3389/fnagi.2014.00284

Bugos, J. A., Perlstein, W. M., McCrae, C. S., Brophy, T. S., \& Bedenbaugh, P. H. (2007). Individualized piano instruction enhances executive functioning and working memory in older adults. Aging Ment Health, 11(4), 464-471. doi: 10.1080/13607860601086504

Chen, A. G., Yan, J., Yin, H. C., Pan, C. Y., \& Chang Y. K. (2014). Effects of acute aerobic exercise on multiple aspects of executive function in preadolescent children. Psychology of Sport and Exercise, 15(6), 627-636. doi: 10.1016/j.psychsport.2014.06.004

Diaz Abrahan, V., Shifres, F., \& Justel, N. (2019). Cognitive benefits from a musical activity in older adults. Frontiers in Psycbology, 10, 652. doi: 10.3389/fpsyg.2019.00652 
Díaz, F. \& Pereiro, A. (2018). Neurociencia cognitiva del envejecimiento. Aportaciones y retos. Revista Española de Geriatría y Gerontología, 53(2), 100-104. doi: 10.1016/j.regg.2017.07.002

Erickson, K. I., Voss, M. W., Prakash, R. S., Basak, C., Szabo, A., Chaddock, L., ... Kramer, A. F. (2011). Exercise training increases size of hippocampus and improves memory. Proceedings of the National Academy of Sciences, 108(7), 3017-3022. doi: 10.1073/ pnas. 1015950108

Evans, D. A., Beckett, L. A., Albert, M. S., Hebert, L. E., Scherr, P. A., Funkenstein, H. H., ... Taylor, J. O. (1993). Level of education and change in cognitive function in a community population of older persons. Annals of Epidemiology, 3(1), 71-77. doi: 10.1016/1047-2797(93)90012-S

Fauvel, B., Groussard, M., Mutlu, J., Arenaza-Urquijo, E. M., Eustache, F., Desgranges, B., \& Platel, H. (2014). Musical practice and cognitive aging: Two crosssectional studies point to phonemic fluency as a potential candidate for a use-dependent adaptation. Frontiers in Aging Neuroscience, 6, 227. doi: 10.3389/ fnagi.2014.00227

Ferreri, L., Aucouturier, J.-J., Muthalib, M., Bigand, E., \& Bugaiska, A. (2013). Music improves verbal memory encoding while decreasing prefrontal cortex activity: an fNIRS study. Frontiers in Human Neuroscience, 7, 779. doi: 10.3389/fnhum.2013.00779

Foubert-Samier, A., Catheline, G., Amieva, H., Dilharreguy, B., Helmer, C., Allard, M., ... Dartigues, J. F. (2010). Education, occupation, leisure activities, and brain reserve: A population-based study. Neurobiology of Aging, 33(2), 423.e15-423.e25. doi: 10.1016/j.neurobiolaging.2010.09.023

Getz, G. E. (2011). Brain reserve capacity. In J.S. Kreutzer, J. DeLuca, \& B. Caplan (Eds.) Encyclopedia of Clinical Neuropsychology. New York, NY: Springer.

Grandi, F. \& Tirapu-Ustárroz, J. (2017). Neurociencia cognitiva del envejecimiento: modelos explicativos. Revista Española de Geriatría y Gerontología, 52(6), 326-331. doi: 10.1016/j.regg.2017.02.005

Hanna-Pladdy, B. \& Gajewski, B. (2012). Recent and past musical activity predicts cognitive aging variability: Direct comparison with general lifestyle activities. Frontiers in Human Neuroscience, 6, 198. doi: 10.3389/fnhum.2012.00198

Hanna-Pladdy, B. \& MacKay, A. (2011). The relation between instrumental musical activity and cognitive aging. Neuropsychology, 25(3), 378-386. doi: 10.1037/a0021895

Hayes, S. M., Forman, D. E., \& Verfaellie, M. (2014). Cardiorespiratory fitness is associated with cognitive performance in older but not younger adults. The Journals of Gerontology Series B: Psychological Sciences and Social Sciences, 71(3), 474482. doi: $10.1093 /$ geronb/gbu167

Hogan, C. L., Mata, J., \& Carstensen, L. L. (2013). Exercise holds immediate benefits for affect and cognition in younger and older adults. Psychology and Aging, 28(2), 587-594. doi: 10.1037/a0032634 
Hwang, J., Brothers, R. M., Castelli, D. M., Glowacki, E. M., Chen, Y. T., Salinas, M., ... Calvert, H. G. (2016). Acute high-intensity exercise-induced cognitive enhancement and brain-derived neurotrophic factor in young, healthy adults. Neuroscience Letters, 630(6), 247-253. doi: 10.1016/j.neulet.2016.07.033

Iuliano, E., di Cagno, A., Aquino, G., Fiorilli, G., Mignogna, P., Calcagno, G., ... Di Costanzo, A. (2015). Effects of different types of physical activity on the cognitive functions and attention in older people: A randomized controlled study. Experimental Gerontology, 70(10), 105-110. doi: 10.1016/j.exger.2015.07.008

Justel, N. \& Diaz Abrahan V. (2012). Plasticidad cerebral: Participación del entrenamiento musical. Suma Psicológica, 19(2), 97-108.

Kamijo, K., Hayashi, Y., Sakai, T., Yahiro, T., Tanaka, K., \& Nishihira, Y. (2009). Acute effects of aerobic exercise on cognitive function in older adults. The Journals of Gerontology Series B: Psychological Sciences and Social Sciences, 64B(3), 356-363. doi: $10.1093 /$ geronb/gbp030

Kang, H. J. \& Williamson, J. W. (2013). Background can aid second language learning. Psychology of Music, 42(5), 728-747. doi: 10.1177/0305735613485152

Kimura, K. \& Hozumi, N. (2012). Investigating the acute effect of an aerobic dance exercise program on neuro-cognitive function in the elderly. Psychology of Sport and Exercise, 13(5), 623-629. doi: 10.1016/j.psychsport.2012.04.001

Langlois, F., Vu, T. T. M., Chassé, K., Dupuis, G., Kergoat, M. J., \& Bherer, L. (2012). Benefits of physical exercise training on cognition and quality of life in frail older adults. Journals of Gerontology Series B: Psychological Sciences and Social Sciences, 68(3), 400-404. doi: 10.1093/geronb/gbs069

Lautenschlager, N. T., Cox, K. L., Flicker, L., Foster, J. K., van Bockxmeer, F. M., Xiao, J., ... Almeida, O. P. (2008). Effect of physical activity on cognitive function in older adults at risk for Alzheimer disease: a randomized trial. JAMA: The Journal of the American Medical Association, 300(9), 1027-1037. doi: 10.1001/jama.300.9.1027

Lee, Y., Chan, M. F., \& Mok, E. (2010). Effectiveness of music intervention on the quality of life of older people. Journal of Advanced Nursing, 66(12), 2677-2687. doi: $10.1111 / \mathrm{j} .1365-2648.2010 .05445 . x$

Lee, T. M. C., Wong, M. L., Lau, B. W. M., Lee, J. C. D., Yau, S. Y., \& So, K. F. (2014). Aerobic exercise interacts with neurotrophic factors to predict cognitive functioning in adolescents. Psychoneuroendocrinology, 39(1), 214-224. doi: 10.1016/j. psyneuen.2013.09.019

Mansens, D., Deeg, J. H., \& Comijs, H. C. (2017). The association between singing and/ or playing a musical instrument and cognitive functions in older adults. Aging \& Mental Health, 22(8), 970-977. doi: 10.1080/13607863.2017.1328481

Moussard, A., Bermudez, P., Alain, C., Tays, W., \& Moreno, S. (2016). Life-long music practice and executive control in older adults: An event-related potential study. Brain Research, 1642, 146-153. doi: 10.1016/j.brainres.2016.03.028 
Nagamatsu, L. S., Chan, A., Davis, J. C., Beattie, B. L., Graf, P., Voss, M. W., ... LiuAmbrose, T. (2013). Physical activity improves verbal and spatial memory in older adults with probable mild cognitive impairment: A 6-month randomized controlled trial. Journal of Aging Research, 2013, 861893. doi: 10.1155/2013/861893

Nishiguchi, S., Yamada, S., Tanigawa, T., Sekiyama, K., Kawagoe, T., Suzuki, M., ... Tsuboyama, T. (2015). A 12-week physical and cognitive exercise program can improve cognitive function and neural efficiency in community-dwelling older adults: A randomized controlled trial. Journal of the American Geriatrics Society, 63(7), 1355-1363. doi: $10.1111 /$ jgs. 13481

Nocera, J., Crosson, B., Mammino, K., \& McGregor, K. M. (2017). Changes in cortical activation patterns in language areas following an aerobic exercise intervention in older adults. Neural Plasticity, 2017, 6340302. doi: 10.1155/2017/6340302

Nocera, J. R., Mcgregor, K. M., Hass, C. J., \& Crosson, B. (2015). Spin exercise improves semantic fluency in previously sedentary older adults. Journal of Aging and Physical Activity, 23(1), 90-94. doi: 10.1123/JAPA.2013-0107

Nouchi, R., Taki, Y., Takeuchi, H., Sekiguchi, A., Hashizume, H., Nozawa, T., ... Kawashima, R. (2014). Four weeks of combination exercise training improved executive functions, episodic memory, and processing speed in healthy elderly people: Evidence from a randomized controlled trial. Age, 36(2), 787-799. doi: 10.1007/s11357-013-9588-x

Organización de los Estados Americanos (OEA). (2015). Proyecto de Resolución. Convención Interamericana sobre la protección de los derechos de las personas mayores. Washington, DC: Asamblea General. Cuadragésimo quinto periodo ordinario de sesiones.

Organización Mundial de la Salud. (OMS). (2017). Temas de S alud, Envejecimiento. Recuperado de http://www.who.int/mediacentre/factsheets/fs404/es/

Parbery-Clark, A., Strait, D. L., Anderson, S., Hittner, E., \& Kraus, N. (2011). Musical experience and the aging auditory system: Implications for cognitive abilities and hearing speech in noise. Plos One, 6(5), e18082. doi: 10.1371/journal.pone.0018082

Pontifex, M. B., Hillman, C. H., Fernhall, B., Thompson, K. M., \& Valentini, T. A. (2015). The effect of acute aerobic and resistance exercise on working memory. Medicine and Science in Sport and Exercise, 1(4), 927-934. doi: 10.1249/ MSS.0b013e3181907d69

Radocy, R. E. \& Boyle, J. D. (1988). Psychological Foundations of Musical Behaviour. Sprinfield, IL: Charles C. Thomas.

Román Lapuente, F. \& Sánchez Navarro, J. (1998). Cambios neuropsicológicos asociados al envejecimiento normal. Anales de Psicología, 14(1), 27-43.

Rosano, C., Venkatraman, V. K., Guralnik, J., Newman, A. B., Glynn, N. W., Launer, L., ... Aizenstein, H. (2010). Psychomotor speed and functional brain MRI 2 years after completing a physical activity treatment. Journals of Gerontology - Series A Biological Sciences and Medical Sciences, 65(6), 639-647. doi: 10.1093/gerona/glq038 
Särkämö, T., Tervaniemi, M., Laitinen, S., Forsblom, A., Soinila, S., Mikkonen, M., ... Hietanen, M. (2008). Music listening enhances cognitive recovery and mood after middle cerebral artery stroke. Brain, 131(Pt 3), 866-876. doi: 10.1093/brain/awn013

Särkämö, T., Tervaniemi, M., Laitinen, S., Numminen, A., Kurki, M., Johnson, J. K., ... Rantanen, P. (2014). Cognitive, emotional, and social benefits of regular musical activities in early dementia: randomized controlled study. Gerontologist, 54(4), 634650. doi: 10.1093/geront/gnt100

Särkämö, T., Altenmüller, E., Rodríguez-Fornells, A., \& Peretz, I. (2016). Editorial: Music, Brain, and Rehabilitation: Emerging Therapeutic Applications and Potential Neural Mechanisms. Frontiersin Human Neuroscience, 10,103. doi: 10.3389/ fnhum.2016.00103

Schneider, C. E., Hunter, E. G., \& Bardach, S. H. (2018). Potential cognitive benefits from playing music among cognitively intact older adults: A scoping review. Journal of Applied Gerontology. doi: 10.1177/0733464817751198

Seinfeld, S., Figueroa, H., Ortiz-Gil, J., \& Sanchez-Vives, M. (2013). Effects of music learning and piano practice on cognitive function, mood and quality of life in older adults. Frontiers in Psychology, 1(4), 810. doi: 10.3389/fpsyg.2013.00810

Suzuki, T., Shimada, H., Makizako, H., Doi, T., Yoshida, D., Ito, K., ... Kato, T. (2013). A randomized controlled trial of multicomponent exercise in older adults with mild cognitive impairment. Plos One, 8(4), e61483. doi: 10.1371/journal. pone.0061483

Stern, Y., Arenaza-Urquijo, E., Bartés-Faz, D., Belleville, S., Cantilon, M., Chetelat, G., ... Vuoksimaa, E. (2018). Whitepaper: Defining and investigating cognitive reserve, brain reserve, and brain maintenance. Alzheimers \& Dementia, S1552-5260(18), 33491-5. doi: 10.1016/j.jalz.2018.07.219

Ten Brinke, L. F., Bolanzadeh, N., Ngamatsu, L. S., Hsu, C. L., Davis, J. C., Miran-Khan, K., \& Liu-Ambrose, T. (2015). Aerobic exercise increases hippocampal volume in older women with probable mild cognitive impairment: A 6-month randomized controlled trial. British Journal of Sports Medicine, 49(4), 248-254. doi: 10.1136/ bjsports-2013-093184

Trombetti, A., Hans, M., Herrmann, F.R., Kressing, R. W., Ferrari, S., \& Rizzoli, R. (2011). Effect of music-based multitask training on gait, balance, and fall risk in elderly people: A randomized controlled trial. Archives of Internal Medicine, 171(6), 525-33. doi: 10.1001/archinternmed.2010.446.

Tsai, C. L., Ukropec, J., Ukropcová, B., \& Pai, M. C. (2018). An acute bout of aerobic or strength exercise specifically modifies circulating exerkine levels and neurocognitive functions in elderly individuals with mild cognitive impairment. NeuroImage: Clinical, 17(1), 272-284. doi: 10.1016/j.nicl.2017.10.028

Voelcker-Rehage, C., Godde, B., \& Staudinger, U. M. (2011). Cardiovascular and coordination training differentially improve cognitive performance and neural processing in older adults. Frontiers in Human Neuroscience, 5(3). doi: 10.3389/fnhum.2011.00026 
Wang, C., \& Schlaug, G. (2010). Music making as a tool for promoting brain plasticity across the life span. Neuroscientist, 16(5), 566-577. doi: 10.1177/1073858410377805

Wang, H. X., Xu W., \& Pei J. J. (2012). Leisure activities, cognition and dementia. Biochimica et Biophysica Acta, 1822(3), 482-491. doi: 10.1016/j.bbadis.2011.09.002

Watanabe, D., Savion-Lemieux, T., \& Penhune, V. (2007). The effect of early musical training on adult motor performance: evidence for a sensitive period in motor learning. Experimental Brain Research, 176(2), 332-40. doi: 10.1007/s00221-006-0619-z

White-Schwoch, T., Woodruff. C., Anderson, S., Strait, D., \& Kraus, N. (2013). Older adults benefit from music training early in life: Biological evidence for long-term training-driven plasticity. Journal of Neuroscience, 33(45) 17667-17674. doi: 10.1523/ JNEUROSCI.2560-13.2013

Zatorre, R. J. \& McGill, J. (2005). Music, the food of neuroscience? Nature, 434(7031), 312-315. doi: $10.1038 / 434312 \mathrm{a}$

Zendel, B. R. \& Alain, C. (2012). Musicians experience less age-related decline in central auditory processing. Psychological Aging, 27(2), 410-417. doi: 10.1037/a0024816 